\title{
IMPACTO DO MÉTODO BLW (BABY LED WEANING) NA ALIMENTAÇÃO COMPLEMENTAR DOS BEBÊS - UMA REVISÃO INTEGRATIVA
}

\section{IMPACT OF BLW (BABY LED WEANING) METHOD ON BABY ADDITIONAL FEEDING - AN INTEGRATIVE REVIEW}

\section{Ketlin Luana Gomes da Costa Ramos}

Nutricionista, Faculdade Presidente Antônio Carlos

UNIPAC de Teófilo Otoni, Brasil.

E-mails: ketlinlu.nutri@hotmail.com

Thais Alves Medeiros

Nutricionista, Faculdade Presidente Antônio Carlos

UNIPAC de Teófilo Otoni, Brasil.

E-mails: thaisalvesmedeiros33@hotmail.com

\section{Karine Rodrigues da Silva Neumann}

Nutricionista especialista em Vigilância em Saúde, Faculdade Presidente Antônio Carlos UNIPAC de Teófilo Otoni, Brasil. E-mails: krsnut@yahoo.com.br

\section{Resumo}

O presente estudo tem como objetivo identificar o impacto do método BLW (Baby Led Weaning) como estratégia de alimentação complementar na introdução alimentar dos bebês. A complementação do leite materno a partir dos seis meses é fundamental para atender as necessidades nutricionais do lactente e com isso tem-se discutido o método de introdução alimentar BLW. Porém, como ainda há controvérsias em algumas literaturas, Nutricionistas tem receio em indicar esse método, sendo necessários estudos sobre o tema. Nesse método é ofertado para o bebê alimentos em pedaços cortados na forma de bastões ou tiras e não se utiliza nenhum tipo de talher ou mistura de alimentos na forma pastosa. Foram incluídos neste estudo artigos do período de 2011 a 2018, no idioma inglês, disponível nas bases de dados científicos, totalizando 7 trabalhos. Os resultados mostram que com o método BLW os bebês apresentam maior participação nas refeições da família; não parecem estar mais propensos a engasgos do que bebes que seguem a abordagem tradicional; fortalece o vínculo entre pais e filhos; os bebês aprendem a preferir alimentos mais saudáveis, há maior capacidade de resposta à saciedade regulando a ingestão de alimentos. Porém a controvérsias, pois em um dos artigos o BLW não resultou em IMC mais apropriado do que a alimentação tradicional, já em outro artigo o IMC foi mais 
apropriado, proporcionou maior preocupação dos pais sobre a capacidade dos bebês de se autoalimentarem e houve diferenças entre os dois métodos em relação ao consumo de energia. É importante que mais estudos sejam feitos para comprovar a real eficácia do método, influência na alimentação da criança e definir limitações com maior segurança, contribuindo para sua aplicação com menores riscos. A metodologia aplicada foi uma revisão integrativa da literatura, através de resumos e artigos experimentais sobre o tema em bases científicas.

Palavras-chave: Método BLW; Alimentação Complementar; Bebês

\section{Abstract}

The present study aims to identify the impact of the BLW (Baby Led Weaning) method as a complementary feeding strategy in the babies' feeding introduction. The complementation of breast milk from six months is essential to meet the nutritional needs of the infant and with this the method of BLW food introduction has been discussed. However, as there are still controversies in some literature, Nutritionists are afraid to indicate this method, requiring studies on the subject. In this method, food is offered to the baby in pieces cut in the form of sticks or strips and no type of cutlery or mixture of food is used in a pasty form. This study included articles from the period 2011 to 2018, in English, available in the scientific databases, totaling 7 works. The results show that with the BLW method, babies have a greater participation in family meals; they do not seem to be more prone to choking than babies who follow the traditional approach; strengthens the bond between parents and children; babies learn to prefer healthier foods, there is a greater capacity to respond to satiety by regulating food intake. However, controversy, as in one of the articles, the BLW did not result in a more appropriate BMI than traditional feeding, in another article, the BMI was more appropriate, provided greater concern for parents about the ability of babies to feed themselves and there were differences between both methods in relation to energy consumption. It is important that more studies are done to prove the real effectiveness of the method, influence the child's diet and define limitations with greater safety, contributing to its application with less risk. The applied methodology was an integrative literature review, through abstracts and experimental articles on the subject in scientific bases

Keywords: BLW method; Complementary Food; Babies

\section{Introdução}

No Brasil, o Ministério da Saúde recomenda o aleitamento materno exclusivo até os primeiros seis meses. Após esse período é necessário a alimentação complementar através da introdução de alimentos de forma gradativa.

A alimentação complementar acontece a partir do momento em que o bebê ainda em período de amamentação, passa a ingerir outros tipos de alimentos tendo em vista que a necessidade de nutrientes é maior devido ao seu crescimento e desenvolvimento.

De acordo com a Sociedade Brasileira de Pediatria o momento para iniciar a alimentação complementar é ao sexto mês de vida, pois nessa fase os bebês já estão 
com desenvolvimento neuropsicomotor e digestivos desenvolvidos o suficiente para a introdução alimentar. (LANIGAN; SINGHAL, 2009; SPB, 2012)

A introdução alimentar tem como característica o primeiro contato com os alimentos sólidos, pastosos e líquidos, além do leite materno, dando início a alimentação complementar. (SILVA, 2013).

A complementação do leite materno a partir dos seis meses é fundamental para atender as necessidades nutricionais do lactente e com isso tem-se discutido o método de introdução alimentar Baby Led Weaning (BLW) que em Português significa desmame conduzido pelo bebê, em substituição ao método tradicional.

Porém, como ainda há controvérsias em algumas literaturas, Nutricionistas tem receio em indicar esse método e muitas mães ainda têm medo de utilizá-lo. Sendo assim, faz-se necessário aprofundar estudos sobre o tema.

Dentro desse contexto, o presente estudo tem como objetivo identificar o impacto do método BLW (Baby Led Weaning) como estratégia de alimentação complementar na introdução alimentar dos bebês.

\section{Passos metodológicos}

O presente estudo trata se de uma revisão integrativa pois possibilita reunir e sintetizar conhecimento de grande valia ao tema proposto, visando buscar na literatura qual o posicionamento sobre o método BLW, por meio da utilização de estudos não experimentais e experimentais para atender ao tema investigado. Sendo esta a mais ampla explanação metodológica referente às revisões. (SOUZA; SILVA; CARVALHO, 2010).

Foi realizada uma revisão integrativa em seis etapas: seleção da questão norteadora; definição das características das pesquisas primárias; seleção das pesquisas que compuseram a amostra; análise dos artigos; interpretação dos resultados; e o exame crítico dos achados, com a seguinte questão norteadora: "qual o impacto do método BLW (Baby Led Weaning) na alimentação complementar dos bebês?".

A coleta de dados ocorreu durante os meses de julho a outubro de 2019. Para consolidação do estudo foi realizada seleção de referências nas bases de dados eletrônicos: National Library of Medicine (MEDLINE), Scientific Electronic Library On Line (SCIELO), UNICEUB e Google Acadêmico, além de livro com abordagem em $B L W$, entre outros visando atender a recomendação da literatura de que se busquem diferentes fontes para o levantamento de publicações. "Foram utilizados os termos "Desmame", "desmame liderado por bebê" e "Nutrição", "Alimentação Infantil", "Babyled Weaning”, "BLW", "Alimentação Complementar". 
Foram incluídas neste estudo pesquisas apresentadas em forma de artigo no período de 2011 a abril de 2018, no idioma inglês disponível nas bases de dados gratuitas e dispostos na forma íntegra para o acesso.

É importante ressaltar que os estudos encontrados em mais de uma das bases de dados utilizadas foram considerados somente uma vez, sendo contabilizados na base que apresentou maior número de trabalhos.

A análise dos dados foi realizada de forma descritiva, procedendo-se à categorização dos dados extraídos dos estudos selecionados em grupos temáticos, a partir da identificação de variáveis de interesse e conceitos-chave, conforme proposto em literatura específica acerca de revisão integrativa de literatura.

\section{Alimentação complementar com o método Baby-Led Weanin (BLW)}

A Organização Mundial da Saúde recomenda que os bebês se alimentem exclusivamente de leite materno até os seis meses de idade, momento em que se inicia a alimentação complementar, porém permanecendo a amamentação até aos 24 meses (OMS, 2018).

A necessidade da alimentação complementar se dá à medida que a criança cresce e somente o leite materno não é capaz de suprir mais as necessidades nutricionais que a criança irá apresentar à medida que crescer (Ministério da Saúde; OMS/WHO, 2003). Além disso, o bebê precisa desenvolver as habilidades de comer e experimentar, para que posteriormente a partir dos dois anos passe a se alimentar somente desses alimentos (OMS, 2018).

No processo de introdução alimentar, os pais ou responsáveis devem observar as necessidades da criança em relação à frequência, quantidade e densidade dos alimentos ofertados, tendo em vista que a criança ainda está amamentando.(RAPLEY, 2015 ; MINISTÉRIO DA SAUDE, 2002).

A Sociedade brasileira de Pediatria e o guia disponibilizado pelo Ministério da Saúde, expressão a mesma orientação em relação à introdução alimentar, pois afirmam que a mesma deve ser feita com alimentos em forma de purê e aos poucos, de forma lenta e gradual, ir modificando até se igualar aos alimentos ingeridos pela família, por volta dos 12 meses. A amamentação deve continuar até que a criança atinja dois anos ou mais (MINISTERIO DA SAÚDE 2009;2012;2013).

A alimentação complementar seguida de forma correta garante o aporte nutricional da criança, sem que haja déficit de vitaminas e minerais, importantes no desenvolvimento neuropsicomotor e fisiológico. (MINISTÉRIO DA SAÚDE, 2014). Os alimentos saudáveis introduzidos nessa fase irão garantir o aporte nutricional, sendo fonte de Ferro, Zinco, Vitamina A e calorias. (SPB, 2017). 
É importante ressaltar que a qualidade e as práticas alimentares adequadas nos primeiros anos de vida influenciam positivamente as condições de saúde da criança a curto e longo prazo. (LANIGAN; SINGHAL, 2009).

Alguns autores afirmam que ao intruzir os alimentos aos bebês, são mais adequados alimentos que necessitam de uma colher, sendo estes pastosos e em forma de purê. A partir do momento que há a erupção dos dentes, progredir para pequenos pedaços macios até que o bebê desenvolva a força e resistência na mastigação, lateralidade da língua, importantes para diminuir o risco de asfixia ( BROWN; LEE, 2013).

Atualmente, uma nova abordagem, o Baby-led Weaning (BLW), vem fazendo cada vez mais parte da introdução e complementação alimentar. O mesmo consiste em introduzir os alimentos sólidos na forma de pedaços cortados em forma de tiras e bastões desde o primeiro contato com a alimentação complementar. A colher não entra e não há nenhum tipo de modificação de consistência das preparações (SBP, 2017).

O método consiste em apresentar a criança diferentes texturas dos alimentos, permitindo que o bebê experimente de forma completa cada alimento, sem que haja a modificação da consistência.

Porém, é importante ressaltar que a comida oferecida em forma de bastão deve passar por um processo de amaciamento, para que a criança possa de alimentar ( WRIGHT et al, 2011)

O BLW é uma atitude de confiança demostrada ao bebê acreditando na capacidade dele de se auto alimentar, desde que este esteja pronto para iniciar a alimentação complementar e demonstre isso por meio de sinais de prontidão, termo usado para nomear os sinais demonstrados pelo bebê como sustentação do pescoço, sentar- se sem o auxilio de um adulto e levar brinquedos a boca com precisão de destreza. (RAPLEY; MURKETT, 2017)

A alimentação é de forma manual, com total liberdade, recebendo os mesmos alimentos que os familiares. O bebê participa das refeições sem deixar de amamentar de forma atender as demandas até ser desmamado.

A introdução dos alimentos no BLW é feita de forma progressiva onde durante algumas semanas devem ser oferecido alimentos que possam ser agarrados e depois em forma de punhados como carne e arroz e depois os alimentos que necessitam que eles façam uma pinça com os dedos ou o uso de talher, mas tudo no tempo do bebê, sem a adição de açúcar, sal, grãos, mel, entre outros. (RAPLEY; MURKETT, 2017).

Ao ser exposto a diferentes formas, cores, texturas e sabores puros de diferentes alimentos, o bebê terá assim o desenvolvimento motor e oral: morder, 
mastigar, engolir cada um no seu tempo, além disso, por ser alimentos em pedaços, é um momento de maior participação da família na refeição. (BROWN; LEE, 2013)

\section{Resultados e discussão}

No geral foram encontrados 645 artigos nas diversas bases de dados, que após análise totalizou 7 artigos sendo 5 no PUBMED, 1 no SCIELO e 1 no MEDLINE

Para a realização deste estudo foram utilizados diversos estudos experimentais e não experimentais, utilizando artigos que relatassem sobre o método BLW e o impacto na alimentação complementar dos bebês.

Um estudo com 650 mães com filhos mais novos ou filho único entre 6 e 12 meses de idade residentes em no condado de Swansea, Reino Unido teve como objetivo fazer comparação entre mães que adotaram o método BLW e as que seguiam o método tradicional, em relação a como introduziram e quando. Mais da metade das mães relataram que usaram o método BLW e iniciaram a introdução alimentar mais tarde, relataram que a técnica permitiu mais autonomia ao bebê fazendo o mesmo participar da mesma refeição da família e menor preocupação materna com o processo de desmame. (BROWN; LEE, 2011)

Uma pesquisa foi realizada com 155 pais de bebês com faixa etária de 20 e 78 meses, recrutados pelo laboratório da criança de Nottingham com o intuito de saber a ingestão alimentar de cada um e o impacto no índice de massa corporal. $O$ estudo demonstrou que crianças que utilizaram o método BLW aprenderam a perceber os sinais de saciedade e fome, levando ao um IMC menor e preferência por alimentos mais saudáveis, comparado ao método tradicional. (TOWNSEND; PITCHFORD, 2012)

Com intuito de comparar o comportamento alimentar das crianças de 18 a 24 meses entre os bebês desmamados usando uma abordagem tradicional de desmame e aqueles usando o método BLW, após um ano de seguimento, foi realizado um estudo com 298 mães de bebês entre 18 e 24 meses de idade. Os bebês usando o método BLW, tinham peso corporal médio significativamente menor (em 1,07 kg) apresentado maior saciedade e menor probabilidade de estar acima do peso quando comparados aos do método tradicional, além de serem menos exigentes em relação aos alimentos. De acordo com o estudo o desmame liderado pelo bebê pode trazer resposta positiva, na capacidade de saciedade, e também, em ganho de peso de forma saudável.( BROWN; LEE, 2013)

Um estudo controlado randomizado em dois anos foi realizado com 206 bebês saudáveis com objetivo de determinar qual o impacto do risco de asfixia em bebês que segue a introdução alimentar tradicional e dos que seguem o método BLW. O presente estudo usou a abordagem baby Led Introduction to solids (BLISS), introdução aos 
sólidos guiada pelo bebê, que é o método BLW, porém com orientação profissional. Como resultado, perceberam que bebês que foram inseridos na alimentação complementar pela abordagem conduzida pelo bebê sob orientação, não apresentaram maior risco de engasgo comparado ao da abordagem de introdução alimentar tradicional, embora os alimentos denominados como risco de asfixiem trouxeram preocupação aos pais. (FANGUPO et al, 2016).

Com duração de dois anos, um estudo clínico randomizado foi realizado com 206 bebês no intuito de determinar se uma abordagem conduzida por bebês à alimentação complementar resulta em um menor índice de massa corporal (IMC) do que a tradicional alimentação por colher. Os autores concluíram com o estudo que uma abordagem conduzida por bebês à alimentação complementar, não apresenta melhor auto regulação da energia ingerida e não resultou em IMC mais apropriado do que a alimentação tradicional por colher. (TAYLOR et al., 2017).

Um estudo transversal de comportamentos alimentares e de ingestão alimentar foi realizado com 51 bebês da mesma idade e sexo sendo 25 deles seguindo do método BLW e 26 do método tradicional, com faixa etária de 6 a 8 meses de idade. 0 estudo tinha como objetivo determinar se há diferenças na ingestão de nutrientes e na ingestão de alimento entre os bebês que seguem BLW e aqueles que seguem o método tradicional. Através do estudo pode-se perceber que os lactentes que seguiam o BLW tinham consumo de energia semelhante aos que seguiam o método tradicional e estavam comendo refeições em família com mais regularidade, porém, com o método BLW, houve um maior consumo de gordura total saturada e menor de ferro, zinco e vitamina B12. (MORISON et al., 2018)

Outro estudo randomizado foi realizado com 206 participantes, parte designados para o grupo BLISS. O objetivo era determinar a ingestão e o status de ferro de bebês após o BLW Modificado para evitar a deficiência de ferro (Introdução aos Sólidos; BLISS) e comparar com aqueles bebês que seguem a alimentação tradicional por colher. Foi coletada amostra de sangue por punção venosa em 12 meses com objetivo de determinar as concentrações plasmáticas de ferrina, hemoglobina, receptor solúvel de transferrina, proteína C-reativa e concentrações de glicoproteína ácida e o ferro corporal foi calculado. Não houve muita diferenciação na ingestão de ferro entre os grupos controle e BLISS, nem diferenças significativas na concentração plasmática de ferritina, ferro corporal ou nas reservas de ferro empobrecidas, deficiência funcional de ferro ou anemia ferropriva, aos 12 meses de idade. (DANIELS et al., 2018)

A partir da análise dos textos selecionados, os dados foram organizados em um quadro (Quadro 1), que mostra a distribuição dos estudos incluídos na revisão 
integrativa, de acordo com autores e ano de publicação, título, base de dados, objetivo e impacto do método BLW:

Quadro 1 - Distribuição dos estudos incluídos na revisão integrativa, de acordo com autores e ano de publicação, título, base de dados, objetivo e impacto do método.

\begin{tabular}{|c|c|c|c|c|}
\hline $\begin{array}{c}\text { AUTORES E } \\
\text { ANO DE } \\
\text { PUBLICAÇÃO }\end{array}$ & TÍTULO & $\begin{array}{c}\text { BASE DE } \\
\text { DADOS }\end{array}$ & OBJETIVO & IMPACTO DO MÉTODO \\
\hline $\begin{array}{l}\text { BROWN, A; } \\
\text { LEE, M; } \\
(2011)\end{array}$ & $\begin{array}{l}\text { A } \\
\text { descriptiv } \\
\text { e study } \\
\text { investigati } \\
\text { ng the use } \\
\text { and } \\
\text { nature of } \\
\text { baby-led } \\
\text { weaning } \\
\text { in UK } \\
\text { sample of } \\
\text { mothers. }\end{array}$ & SCIELO & $\begin{array}{l}\text { Comparar mães que } \\
\text { seguiram o BLW e } \\
\text { mães que usaram } \\
\text { desmame convencional. }\end{array}$ & $\begin{array}{l}\text {-Introdução de alimentos } \\
\text { complementares mais } \\
\text { tarde do que os que } \\
\text { seguem uma abordagem } \\
\text { tradicional. } \\
\text { - Maior participação nas } \\
\text { refeições da família e } \\
\text { menos preocupações } \\
\text { maternas com o processo } \\
\text { de desmame. }\end{array}$ \\
\hline $\begin{array}{l}\text { TOWNSEND, } \\
\text { PITCHFORD } \\
2012\end{array}$ & $\begin{array}{l}\text { Baby } \\
\text { knows } \\
\text { best. The } \\
\text { impact of } \\
\text { weaning } \\
\text { style 1on } \\
\text { food } \\
\text { preferenc } \\
\text { es and } \\
\text { body } \\
\text { mass } \\
\text { index in } \\
\text { early } \\
\text { childhood } \\
\text { in a } \\
\text { caseecont } \\
\text { rolled } \\
\text { sample }\end{array}$ & PUBMED. & $\begin{array}{l}\text { Examinar se o método } \\
\text { de desmame liderado } \\
\text { por bebê versus } \\
\text { alimentação tradicional } \\
\text { com colher influência } \\
\text { nas preferências } \\
\text { alimentares e se há } \\
\text { alteração no índice de } \\
\text { massa corporal }\end{array}$ & $\begin{array}{l}\text { - Os bebês desmamados } \\
\text { por meio do BLW } \\
\text { aprenderam a regular sua } \\
\text { ingestão de alimentos e } \\
\text { apresentou menor IMC, } \\
\text { comparado ao método } \\
\text { tradicional. }\end{array}$ \\
\hline $\begin{array}{l}\text { BROWN A; LEE } \\
\text { MD; } \\
\text { (2013) }\end{array}$ & $\begin{array}{l}\text { Early } \\
\text { influences } \\
\text { on child } \\
\text { satiety } \\
\text { responsiv } \\
\text { eness: the } \\
\text { role of } \\
\text { weaning } \\
\text { style. }\end{array}$ & PUBMED & $\begin{array}{l}\text { Comparar } \\
\text { comportamento } \\
\text { alimentar dos bebês de } \\
18 \text { a } 24 \text { meses de idade } \\
\text { entre os que seguiam } \\
\text { uma abordagem } \\
\text { tradicional e o estilo } \\
\text { BLW, conduzido pelo } \\
\text { bebê. }\end{array}$ & $\begin{array}{l}\text {-Maior capacidade de } \\
\text { resposta à saciedade e } \\
\text { trajetórias saudáveis de } \\
\text { ganho de peso em bebês. }\end{array}$ \\
\hline $\begin{array}{l}\text { FANGUPO, L. } \\
\text { J. et al. } \\
(2016)\end{array}$ & $\begin{array}{l}\text { A Baby- } \\
\text { Led } \\
\text { Approach } \\
\text { to Eating } \\
\text { Solids and } \\
\text { Risk of }\end{array}$ & PUBMED & $\begin{array}{l}\text { Determinar o impacto } \\
\text { de uma abordagem } \\
\text { liderada por bebês na } \\
\text { alimentação } \\
\text { complementar } \\
\text { enaasqo e asfixia da }\end{array}$ & $\begin{array}{l}\text {-Não houve propensão a } \\
\text { engasgos no bebê quando } \\
\text { a mãe é aconselhada } \\
\text { sobre como minimizar o } \\
\text { risco de asfixia. }\end{array}$ \\
\hline
\end{tabular}




\begin{tabular}{|c|c|c|c|c|}
\hline & Choking. & & criança. & \\
\hline $\begin{array}{l}\text { TAYLOR RW; et } \\
\text { al. } \\
(2017)\end{array}$ & $\begin{array}{l}\text { Effect of a } \\
\text { Baby-Led } \\
\text { Approach } \\
\text { to } \\
\text { Complem } \\
\text { entary } \\
\text { Feeding } \\
\text { on Infant } \\
\text { Growth } \\
\text { and } \\
\text { Overweig } \\
\text { ht: A } \\
\text { Randomiz } \\
\text { ed Clinical } \\
\text { Trial. }\end{array}$ & $\begin{array}{l}\text { NATIONAL } \\
\text { LIBRARY } \\
\text { OF } \\
\text { MEDICINE } \\
\text { NATIONAL } \\
\text { INSTITUTE } \\
\text { S OF } \\
\text { HEALTH }\end{array}$ & $\begin{array}{l}\text { Determinar se uma } \\
\text { abordagem liderada por } \\
\text { bebês à alimentação } \\
\text { complementar resulta } \\
\text { em um menor índice de } \\
\text { massa corporal (IMC) } \\
\text { do que a tradicional } \\
\text { alimentação por colher. }\end{array}$ & $\begin{array}{l}\text { - O método BLW não } \\
\text { apresentou } \\
\text { autorregulação da energia } \\
\text { ingerida pelos bebês e não } \\
\text { resultou em IMC mais } \\
\text { apropriado do que a } \\
\text { alimentação tradicional por } \\
\text { colher. }\end{array}$ \\
\hline $\begin{array}{l}\text { Morison BJ, et } \\
\text { al. } \\
2018\end{array}$ & $\begin{array}{l}\text { How } \\
\text { different } \\
\text { are baby- } \\
\text { led } \\
\text { weaning } \\
\text { and } \\
\text { conventio } \\
\text { nal } \\
\text { compleme } \\
\text { ntary } \\
\text { feeding? } \\
\text { A cross- } \\
\text { sectional } \\
\text { study of } \\
\text { infants } \\
\text { aged 6-8 } \\
\text { months }\end{array}$ & PUBMED & $\begin{array}{l}\text { Determinar se há } \\
\text { diferenças na ingestão } \\
\text { de nutrientes e na } \\
\text { ingestão de alimento } \\
\text { entre os bebês que } \\
\text { seguem BLW e aqueles } \\
\text { que seguem o método } \\
\text { tradicional. }\end{array}$ & $\begin{array}{l}\text { - Os lactentes que seguiam } \\
\text { o BLW tinham consumo de } \\
\text { energia semelhante aos } \\
\text { que seguiam o método } \\
\text { tradicional e estavam } \\
\text { comendo refeições em } \\
\text { família com mais } \\
\text { regularidade } \\
\text { - Com o método BLW há } \\
\text { um maior consumo de } \\
\text { gordura total saturada e } \\
\text { menor de ferro, zinco e } \\
\text { vitamina B12. }\end{array}$ \\
\hline $\begin{array}{l}\text { DANIELS L, } \\
\text { TAYLOR RW, } \\
\text { WILLIAMS SM, } \\
\text { ET AL. } \\
2018\end{array}$ & $\begin{array}{l}\text { Impact of } \\
\text { a modified } \\
\text { version of } \\
\text { babyled } \\
\text { weaning } \\
\text { on iron } \\
\text { intake and } \\
\text { status: a } \\
\text { randomise } \\
\text { d } \\
\text { controlled } \\
\text { trial }\end{array}$ & PUBMED & $\begin{array}{l}\text { Determinar a ingestão e } \\
\text { o status de ferro de } \\
\text { bebês após o BLW } \\
\text { Modificado para evitar a } \\
\text { deficiência de ferro } \\
\text { (Introdução aos Sólidos; } \\
\text { BLISS) e comparação } \\
\text { com aqueles de bebês } \\
\text { que seguem a a } \\
\text { alimentação tradicional } \\
\text { por colher. }\end{array}$ & $\begin{array}{l}\text { - Uma abordagem liderada } \\
\text { por bebês pode ser usada } \\
\text { sem afetar negativamente } \\
\text { o status do ferro desde que } \\
\text { seja bem orientada }\end{array}$ \\
\hline
\end{tabular}

Por meio dos estudos é possível perceber que existem algumas vantagens ao utilizar o método BLW como regulação da saciedade e controle da fome, menor stress ao se alimentar, tanto dos pais quanto do bebê, não apresentam maior propensão ao engasgo desde que seja orientado, porém existem também alguns artigos que 
mostram que com o método pode-se obter resultados diferentes em relação ao consumo de energia, e IMC.

Revista Multidisciplinar do Nordeste Mineiro, v...., n..., p. 01-X, 2018/02 ISSN 2178-6925 / doi.org/10.17648/2178-6925

\section{Considerações finais}

A alimentação complementar é essencial após o sexto mês, mantendo a amamentação, porém ainda existem dúvidas de como deve ser a ingestão alimentar que garanta as necessidades nutricionais da criança tanto.

A utilização do método Baby Led Weaning (BLW) está crescendo a cada dia, mas a falta de informações sobre a mesmo influência diretamente na escolha do método e na sua aplicação de forma correta.

Apesar de suas vantagens o método não deve ser adotado para todas e quaisquer crianças, uma vez que o tempo de evolução física necessária para aplicar o BLW varia entre 6 e 7 meses. A depender do desenvolvimento da criança, podem-se alternar entre os dois métodos, fazendo uso do método convencional nas principais refeições e do método BLW nas secundárias para estimular o consumo e familiarizar o bebê com essa forma de alimentação.

É responsabilidade do Nutricionista juntamente com outros profissionais da saúde avaliar individualmente o paciente para depois decidir qual a melhor conduta dietoterápica que deverá ser aplicada, levando em consideração características do ambiente familiar, além das necessidades nutricionais do bebê.

Os pais que optarem pelo BLW devem ser orientados pelo Nutricionista sobre a oferta de uma maior variedade de alimentos ricos em diversos tipos de nutrientes e minerais, como ferro, zinco e vitamina B12 para alcançar as necessidades do bebê

A viabilidade, os riscos e os benefícios do BLW como uma abordagem à alimentação infantil só podem ser determinados em um estudo em que bebês e suas famílias são randomizados para seguir o BLW, e seus resultados comparados aos de um grupo controle seguindo práticas alimentares padrão. Sendo assim ainda há a necessidade de mais pesquisa para afirmar que o método é a melhor estratégia de alimentação complementar.

\section{Referências}


BROWN A, LEE M. Maternal control of child feeding during the weaning period: differences between mothers following a baby-led or standard weaning approach.; v.15 e.8 p. 1265 -1271. Novembro 2011.

BROWN A; LEE MD; Early influences on child satiety responsiveness: the role of weaning style. Rev. Pediatric Obesity , v. 10 n.1 p.57-66, Reino Unido,2013 Disponível em:< https://onlinelibrary.wiley.com/doi/epdf/10.1111/j.20476310.2013.0020. >.Acesso em: 20 Nov 2019.

CAMASMIE, L.E.M. Introdução Alimentar: Comparação Entre O Método Tradicional E O Baby-Led Weaning (BLW). CONIC-SEMESP, São Paulo,2018, Disponível em:< http://conic-semesp.org.br/anais/files/2018/trabalho-1000002654.pdf.< Acesso em 12 out 2019

DANIELS L et al. Impact of a modified version of babyled weaning on iron intake and status: a randomised controlled trial. BMJ Open v.8 e.6 maio 2018 . Disponível em:> https://www.ncbi.nlm.nih.gov/pmc/articles/PMC6020950/pdf/bmjopen-2017019036.pdf.< acesso em: 20 out.2019.

FANGUPO LJ, et al. A Baby-Led Approach to Eating Solids and Risk of Choking.

Pediatrics v.138 p.4 outubro 2016. Disponível

em:>https://pediatrics.aappublications.org/content/pediatrics/138/4/e20160772.full.pdf. $<$ Acesso em jul 2019

LANIGAN J, SINGHAL A. Early nutrition and long-term health: a pratical approach. Proceedings of the Nutrition Society v.68 n.4 p. 422-9, Nov 2009, Disponível em: $<$ https://www.cambridge.org/core/services/aop-cambridgecore/content/view/0A17AE2467D5BB634C436D1E1940C760/S002966510999019Xa.p df/early_nutrition_and_longterm_health_a_practical_approach.pdf.> .Acesso em: 20 jul.2019.

MINISTÉRIO DA SAÚDE. Dez passos para uma alimentação saudável: guia alimentar para crianças menores de dois anos: um guia para o profissional da saúde na atenção básica / Ministério da Saúde. 2. ed. Brasília: Ministério da Saúde; 2013.

MINISTÉRIO DA SAÚDE. Guia alimentar para a população brasileira. 2014.

MINISTÉRIO DA SAÚDE. Organização Pan-Americana da Saúde: Guia Alimentar para a crianças menores de dois anos. Brasília: Pan-americana da Saúde; 2002.

MINISTÉRIO DA SAÚDE. Saúde da criança: crescimento e desenvolvimento. 2012.

MINISTÉRIO DA SAÚDE. Saúde da Criança: nutrição infantil: aleitamento materno e alimentação complementar. Brasília: Editora do Ministério da Saúde; 2009.

MORISON, B.J, et al. How different are baby-led weaning and conventional complementary feeding? A cross-sectional study of infants aged 6-8 months. Jornal BMJ Open, 2018; v.6 ed 5. Disponível em:< https://bmjopen.bmj.com/content/bmjopen/6/5/e010665.full.pdf>. Acesso em 14 julho 2019

OMS. Organização Mundial da Saúde. Folha informativa - Alimentação saudável. 2018.

OMS/WHO. World Health Organization. Global strategy for infant and young child feeding. Geneva: World Health Organization; 2003. 
<https://journals.sagepub.com/doi/pdf/10.1177/1941406415575931.< Acesso em 10 out 2019

RAPLEY G, MURKETT T. Baby-led weaning: o desmame guiado pelo bebê. São Paulo: Timo; 2017.

SBP. SOCIEDADE BRASILEIRA DE PEDIATRIA. Manual de orientação para a alimentação do lactente, do pré-escolar, do escolar, do adolescente e na escola/Sociedade Brasileira de Pediatria. Departamento de Nutrologia, 3a. ed. Rio de Janeiro, RJ: SBP, 2012.

SBP.Sociedade Brasileira de Pediatria. Guia Prático de Atualização Departamento Científico de Nutrologia: A Alimentação Complementar e o Método BLW (Baby-Led Weaning). Sociedade Brasileira de Pediatria.2017 Disponível em:

>https://www.sbp.com.br/fileadmin/user_upload/19491c-GP___AlimCompl_-

_Metodo_BLW.pdf.<.Acesso em 15 out.2019.

SILVA, M. C. S. Tratado de alimentação, nutrição e dietoterapia. Sandra Maria Chemin Seabra da Silva, Joana D’Arc Pereira Mura. 2.ed. [Reimpr.]. São Paulo: Roca, 2013..

SOUZA, M. T.; SILVA, M. D.; CARVALHO, R. Revisão integrativa: o que é e como fazer. Rev. Eisntein. 2010; v.8, p.1102-6. Disponível em <http://www.astresmetodologias.com/material/O_que_e_RIL.pdf>. Acesso em 25 Out. 2019

TAYLOR, R. W. et al. Effect of a Baby-Led Approach to Complementary Feeding on 36 Infant Growth and Overweight. A Randomized Clinical Trial. JAMA Pediatrics, v.171 n.9 p. 838-846. Disponível em:>https://byebyepurees.com/wpcontent/uploads/2017/09/34.-Effect_of_a_baby-led_approach_to.pdf.<. Acesso em jul 2019

TOWNSEND E; PITCHFORD NJ; Baby knows best? The impacto of weaning style on food preferences and body mass index in early childhood in a case - controlled sample. BMJ Open.v.2.n.1 Reino Unido, 2012.Disponível em:

>https://bmjopen.bmj.com/content/bmjopen/2/1/e000298.full.pdf.<. Acesso em agos 2019.

WRIGHT CM, et al. Is baby-led weaning feasible? When do babies first reach outfor and eat finger foods?. v.7 n.1 p. 27-33.2011 Disponível em:

>https://onlinelibrary.wiley.com/doi/epdf/10.1111/j.1740-8709.2010.00274.x.<Acesso em: 12 out 2019 